\title{
Weighted Multirate q-Markov Cover Identification Using PRBS - An Application to Engine Systems
}

\author{
G. GEORGE ZHU \\ Cummins Engine Company, 1900 Mckinley Ave., MC 50197, \\ Columbus, IN 47201, USA
}

(Received 9 August 1999)

The q-Markov COVariance Equivalent Realization (q-Markov Cover) method for identification uses either pulse, white noise or PRBS (Pseudo-Random Binary Signal) as test excitation. This paper extended the q-Markov Cover using PRBS to the weighted multirate case, that is, the sample rate of the PRBS signal is different from the system output one. Then, the multirate PRBS q-Markov Cover is applied to identify a diesel engine model from the fuel command input to the engine speed output. The identified engine model has order of two and approximates the pure fuel system time delay using a first-order transfer function with a non-minimum phase numerator. Finally, the identified engine model was successfully used for designing engine idle speed governor and obtained satisfactory performance in the first try.

Keywords: System identification; Pseudo-random binary signal; Control systems

\section{INTRODUCTION}

The q-Markov COVariance Equivalent Realization (q-Markov Cover) theory was originally developed for model reduction. The reduced order model obtained using q-Markov Cover preserves the first $q$ Markov and covariance parameters of the original system. The parameterization of all q-Markov Covers for both continuous and discrete systems is addressed in Ref. [1]. The realization of all q-Markov Covers from the input/output data of discrete systems is useful for identification; see Ref. [2]. This algorithm generates all q-Markov 
Covers with only $q$ Markov parameters and $q$ covariance parameters. This data set requires both pulse experiments to obtain the Markov parameters and white noise experiments to get the covariance parameters. The deterministic approach using only pulse experimental data and the stochastic approach using only white noise experimental data to generate the $q$ Markov parameters and covariance parameters are described in Ref. [3]. Using the results in Ref. [3], a q-Markov Cover of a linear discrete system can be found by using experimental data from either pulse or white noise input. The q-Markov Cover identification using experimental data from Pseudo-Random Binary Signal (PRBS) (see Refs. [4,5]) is presented in Ref. [6] to replace the white noise version algorithm due to the easy implementation of PRBS. These signal can be generated using simple shift register circuits and it is very practical for engineering applications.

The extension of the single rate q-Markov Cover to the multirate case is motivated by its application to the diesel engine system to obtain a linearized transfer function from commanded fueling $\left(\mathrm{mm}^{3} /\right.$ stroke) to engine speed (RPM) at $100 \mathrm{~Hz}$ sample rate. For a six-cylinder fourstroke diesel engine, the engine firing (or fuel injection) frequency is $30 \mathrm{~Hz}$ at $600 \mathrm{RPM}$. The PRBS fuel excitation sampled at more than $30 \mathrm{~Hz}$ will not be executed by the fuel injectors, providing a badly identified model due to poor correlation between the fuel command input and the engine speed output. To improve the system identification for diesel engine applications using PRBS q-Markov Cover, it is necessary to have a q-Markov Cover capable of using the input and output data sampled at different frequencies. This paper provides a q-Markov Cover theory that allows the input PRBS sampled at a frequency slower than the system output sample rate at which the linear model is constructed.

A Matlab Graphic User Interface (GUI) has been written to make the multirate PRBS q-Markov Cover system identification tool easy to use. The system identification GUI displays the input PRBS and output response, along with the model order selection plot. This makes it convenient to study the relationship between identification error and the model order selection.

The paper is organized as follows. In Section II, the PRBS signals and its multirate sampling scheme are discussed. Section III extends the single rate PRBS q-Markov Cover theory to a weighted multirate 
version. An application example to diesel engine identification is provided in Section IV, and the last section adds some conclusions.

\section{PRBS AND ITS STOCHASTIC PROPERTIES}

\section{II.A. PRBS Used for q-Markov Cover}

The most commonly used PRBSs are based on maximum length sequences (called $m$-sequences) for which the length of the PRBS signals is $m=2^{n}-1$, where $n$ is an integer called the order of the PRBS. Let $z^{-1}$ represent a delay operator, and define $\hat{p}\left(z^{-1}\right)$ to be the polynomials

$$
\hat{p}\left(z^{-1}\right)=a_{n} z^{-(n-1)} \oplus \cdots \oplus a_{2} z^{-1} \oplus a_{1},
$$

where $a_{i}$ is either $a>0$ or $-a$, and for any number $a \oplus a$ obeys,

$$
a \oplus a=-a=-a \oplus-a, \quad a \oplus-a=a=-a \oplus a .
$$

The following table (Table I) provides the selection of coefficients $a_{i}$ $(i=1,2, \ldots, n)$ that guarantees $\hat{p}\left(z^{-1}\right)$ to be a maximum sequence.

The coefficients of the maximum polynomials with order between 12 and 34 can be found in Ref. [4]. The PRBS with magnitude $a$ can be generated by the following formula:

$$
\hat{u}\left[(k+1) T_{\mathrm{p}}\right]=\hat{p}\left(z^{-1}\right) \hat{u}\left(k T_{\mathrm{p}}\right), \quad k=0,1,2, \ldots,
$$

TABLE I Coefficients for maximum sequences

\begin{tabular}{lcc}
\hline $\begin{array}{l}\text { Order of } \\
\text { polynomial } n\end{array}$ & $\begin{array}{c}\text { Period of the } \\
\text { sequence } m\end{array}$ & $\begin{array}{c}\text { Non-negative } \\
\text { coefficients } a_{i}\end{array}$ \\
\hline 2 & 3 & $a_{1}, a_{2}$ \\
3 & 7 & $a_{2}, a_{3}$ \\
4 & 15 & $a_{3}, a_{4}$ \\
5 & 31 & $a_{3}, a_{5}$ \\
6 & 63 & $a_{5}, a_{6}$ \\
7 & 127 & $a_{4}, a_{7}$ \\
8 & 255 & $a_{2}, a_{3}, a_{4}, a_{8}$ \\
9 & 511 & $a_{5}, a_{9}$ \\
10 & 1023 & $a_{7}, a_{10}$ \\
11 & 2047 & $a_{9}, a_{11}$ \\
\hline
\end{tabular}


where $\hat{u}(0)=a$ and $\hat{u}\left(-T_{\mathrm{p}}\right)=\hat{u}\left(-2 T_{\mathrm{p}}\right)=\cdots=\hat{u}\left[-(n-1) T_{\mathrm{p}}\right]=-a$, $T_{\mathrm{p}}$ is the sample rate used to generate the PRBS, and $a$ is the magnitude of the PRBS signal. The inverse PRBS, used for q-Markov Cover, is constructed by

$$
u\left(k T_{\mathrm{p}}\right)=s(k) \oplus \hat{u}\left(k T_{\mathrm{p}}\right), \quad s(k)=\left\{\begin{array}{r}
a, k \text { even } \\
-a, k \text { odd }
\end{array}\right.
$$

It is clear after some analysis that $u(k)$ has a period $2 m$ and $u(k)=$ $-u(k+m)$. The mean of the inverse PRBS is

$$
m_{u}=E_{2 m} u\left(k T_{\mathrm{p}}\right)=\frac{1}{2 m} \sum_{i=0}^{2 m-1} u\left(k T_{\mathrm{p}}\right)=0
$$

and the autocorrelation of $u(k)$ is

$$
\begin{aligned}
R_{u u}\left(\tau T_{\mathrm{p}}\right)= & E_{2 m} u\left[(k+\tau) T_{\mathrm{p}}\right] u^{\mathrm{T}}\left(k T_{\mathrm{p}}\right)=\frac{1}{2 m} \sum_{i=0}^{2 m-1} u\left[(k+\tau) T_{\mathrm{p}}\right] u^{\mathrm{T}}\left(k T_{\mathrm{p}}\right) \\
= & \left\{\begin{array}{cl}
a^{2}, & \tau=0 \\
-a^{2}, & \tau=m \\
-\frac{a^{2}}{m}, & \tau \text { even } \\
\frac{a^{2}}{m}, & \tau \text { odd }
\end{array}\right.
\end{aligned}
$$

In the rest of this paper, the term "PRBS" is used to represent the inverse PRBS.

\section{II.B. PRBS Sampled at Higher Rate than its Generation Rate}

Consider the situation that the PRBS is generated at the sample rate $1 / T_{\mathrm{p}}$ and the signal is sampled at a higher rate $1 / T$ than its generation rate. Assume that the ratio of two sample rates is an integer $n_{\mathrm{r}}$ defined as follows:

$$
n_{\mathrm{r}}=\frac{T_{\mathrm{p}}}{T}
$$


The period number of the PRBS signal sampled at $1 / T$ is $2 n_{\mathrm{r}} m$, and the mean of the signal is zero computed by the following equation:

$$
m_{u}^{\mathrm{T}}=E_{2 n_{\mathrm{r}} m} u(k T)=\frac{1}{2 n_{\mathrm{r}} m} \sum_{i=0}^{2 n_{\mathrm{r}} m-1} u(k T)=0 .
$$

However, the autocorrelation is quite different from the situation that the PRBS is sampled at its generation rate. In this case, the autocorrelation is defined by

$$
R_{u u}(\tau T)=E_{2 n_{\mathrm{r}} m} u[(k+\tau) T] u^{\mathrm{T}}(k T)=\frac{1}{2 n_{\mathrm{r}} m} \sum_{i=0}^{2 n_{\mathrm{r}} m-1} u[(k+\tau) T] u^{\mathrm{T}}(k T)
$$

and equals to

$$
\begin{aligned}
& R_{u u}(\tau T) \\
& = \begin{cases}\left(n_{\mathrm{r}}-\bar{\tau}\right) a^{2}+\bar{\tau} \frac{a^{2}}{m}, & 0 \leq \tau<n_{\mathrm{r}} \text { or } n_{\mathrm{r}} m \leq \tau<n_{\mathrm{r}}(m+1), \\
-\bar{\tau} a^{2}-\left(n_{\mathrm{r}}-\bar{\tau}\right) \frac{a^{2}}{m}, & n_{\mathrm{r}}(m-1) \leq \tau<n_{\mathrm{r}} m \text { or } n_{\mathrm{r}}(2 m-1) \leq \tau<2 n_{\mathrm{r}} m, \\
-\left(n_{\mathrm{r}}-2 \bar{\tau}\right) \frac{a^{2}}{m}, & \text { otherwise if } \tau \text { even, } \\
\left(n_{\mathrm{r}}-2 \bar{\tau}\right) \frac{a^{2}}{m}, & \text { otherwise if } \tau \text { odd, }\end{cases}
\end{aligned}
$$

where $\bar{\tau}=\tau-$ fix $\left(t / n_{\mathrm{r}}\right) n_{\mathrm{r}}$ and fix[ $[\cdot]$ is a round-off operator which takes integer portion of $[\cdot]$. Figure 1 is an autocorrelation plot of a 5 th order PRBS with unit magnitude sampled three times faster than its generation sample rate. When $\tau$ is between -2 and 2, the autocorrelation is greater than one. The absolute value of the rest of the autocorrelation is less than $3 / 31$ (that is $n_{\mathrm{r}} a^{2} / m$ ) when the absolute value of $\tau$ is greater than 2 and less than $n_{\mathrm{r}}(m-1)=90$. Note that when the order of the PRBS increases, $n_{\mathrm{r}} a^{2} / m$ reduces rapidly. For example, for 10th order PRBS with unit magnitude and $n_{\mathrm{r}}=3$, $n_{\mathrm{r}} a^{2} / m=0.00293$ which is less than 0.3 percent of the autocorrelation when $\tau \in[-2,2]$. This property is very important for applying PRBS to q-Markov Cover identification. 


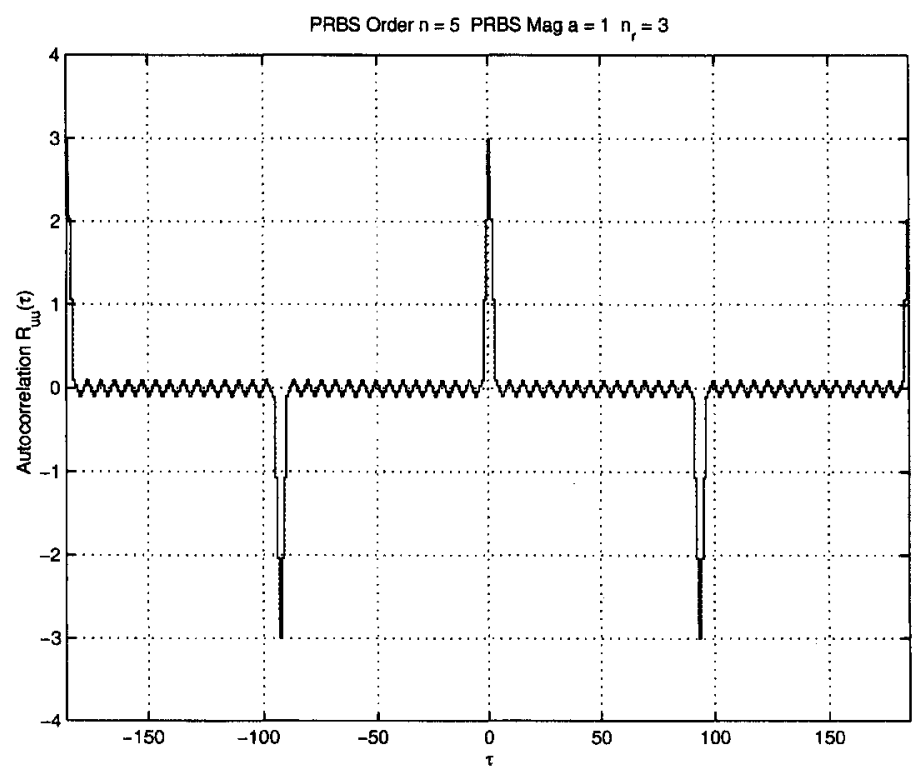

FIGURE 1 Autocorrelation of PRBS sampled faster than its generation rate. (See Color Plate I at the back of the issue.)

\section{WEIGHTED MULTIRATE q-MARKOV COVER USING PRBS}

Consider the discrete asymptotically stable system

$$
\begin{aligned}
x(k+1) & =A x(k)+D w(k), \\
y(k) & =C x(k)+H w(k),
\end{aligned}
$$

where $w \in \mathfrak{R}^{n_{w}}, x \in \mathfrak{R}^{n_{x}}$ and $y \in \mathfrak{R}^{n_{y}}$ are the input, state, and output vectors. Suppose that $X$ is the solution of the following Lyapunov equation:

$$
X=A X A^{\mathrm{T}}+D W D^{\mathrm{T}},
$$

where $W$ is the covariance matrix of the white noise input $w$. The Markov parameters $H_{i}$ and covariance parameters $R_{i}(i=0,1,2, \ldots)$ of (III-1) are defined by

$$
\begin{aligned}
H_{0} & =H, \quad H_{i}=C A^{i-1} D, i=1,2, \ldots, \\
R_{i} & =C A^{i} X C^{\mathrm{T}}+H_{i} W H^{\mathrm{T}}, i=0,1,2, \ldots
\end{aligned}
$$




\section{III.A. System Responses and its Autocorrelation}

Use the same approach as Ref. [6], let $x(k, I)$ denote the state response of (III-1) with $e_{I} u$ as input, where $e_{I}$ is the Ith standard base vector in $\mathfrak{R}^{n_{w}}$ and $u$ is the PRBS sampled $n_{\mathrm{r}}$ times faster than its generation sample rate with a period number $m$. Since the PRBS is a periodic signal with period number $2 n_{\mathrm{r}} m$, the steady state response of the linear system (III-1) will also be periodic with the same period number as the PRBS. For any integer $p>0$. Let $x\left(2 n_{\mathrm{r}} m p+k\right)$ represent the state response of (III-1) at time $\left(2 n_{\mathrm{r}} m p+k\right) T$ and for $0 \leq k<2 n_{\mathrm{r}} m$ define the steady state response with input $e_{I} u$ as

$$
\begin{aligned}
x_{s}^{I}(k) & =\lim _{p \rightarrow \infty} x^{I}\left(2 n_{\mathrm{r}} m p+k\right) \\
& =A^{k} \sum_{j=0}^{\infty} A^{2 n_{\mathrm{r}} m j} \sum_{i=1}^{2 n_{\mathrm{r}} m-1} A^{2 n_{\mathrm{r}} m-1-i} D e_{I} u(i)+\sum_{i=0}^{k-1} A^{k-1-i} D e_{I} u(i) .
\end{aligned}
$$

Assume stable system (III-1), leading

$$
x_{s}^{I}(k+i)=A^{k} x_{s}^{I}(k)+\sum_{j=0}^{i-1} A^{i-1-j} D e_{I} u(j), \quad i=0,1, \ldots, q-1 .
$$

Using (III-1) and (III-5) we can have the following relationship:

$$
Q_{q} y_{q}^{I}(k)=Q_{q} O_{q} x_{s}^{I}(k)+Q_{Q} H_{q} w_{q}^{I}(k),
$$

where $Q_{q}$ is a block diagonal matrix with $q$ blocks of positive definite output weighting matrix $Q$, and

$$
\begin{gathered}
y_{q}^{I}(k)=\left[\begin{array}{c}
y_{s}^{I}(k) \\
y_{s}^{I}(k+1) \\
\vdots \\
y_{s}^{I}(k+q-1)
\end{array}\right], \quad w_{q}^{I}(k)=\left[\begin{array}{c}
e_{I} u(k) \\
e_{I} u(k+1) \\
\vdots \\
e_{I} u(k+q-1)
\end{array}\right], \\
O_{q}=\left[\begin{array}{c}
C \\
C A \\
\vdots \\
C A^{q-1}
\end{array}\right], \quad H_{q}=\left[\begin{array}{cccc}
H_{0} & 0 & \ldots & 0 \\
H_{1} & H_{0} & \ldots & 0 \\
\vdots & \vdots & \ddots & \vdots \\
H_{q-1} & H_{q-2} & \ldots & H_{0}
\end{array}\right]
\end{gathered}
$$


Taking the expectation over one PRBS period from both sides of Eq. (III-6) yields

$$
Q_{q} R_{q}^{I} Q_{q}^{\mathrm{T}}=Q_{q} O_{q} X^{I} O_{q}^{\mathrm{T}} Q_{q}^{\mathrm{T}}+Q_{q} H_{q} W_{q}^{I} H_{q}^{\mathrm{T}} Q_{q}^{\mathrm{T}}+Q_{q} \Delta_{q}^{I} Q_{q}^{\mathrm{T}},
$$

where for any $q<n_{\mathrm{r}} m$, matrix $R_{q}^{I}$ has Toeplitz structure. Define

$$
R_{q}=\sum_{I=1}^{n_{W}} R_{q}^{I}, \quad X=\sum_{I=1}^{n_{\aleph}} X^{I}, \quad W_{q}=\sum_{I=1}^{n_{w}} W_{q}^{I}, \quad \Delta_{q}=\sum_{I=1}^{n_{w}} \Delta_{q}^{I} .
$$

Then for any $q<n_{\mathrm{r}} m$ we have

$$
Q_{q} R_{q} Q_{q}^{\mathrm{T}}=Q_{q} O_{q} X O_{q}^{\mathrm{T}} Q_{q}^{\mathrm{T}}+Q_{q} H_{q} W_{q} H_{q}^{\mathrm{T}} Q_{q}^{\mathrm{T}}+Q_{q} \Delta_{q} Q_{q}^{\mathrm{T}},
$$

where

$$
\begin{aligned}
& R_{q}=\left[\begin{array}{cccc}
R_{y y}(0) & R_{y y}(1) & \ldots & R_{y y}(q-1) \\
R_{y y}(1) & R_{y y}(0) & \ldots & R_{y y}(q-2) \\
\vdots & \vdots & \ddots & \vdots \\
R_{y y}(q-1) & R_{y y}(q-2) & \ldots & R_{y y}(0)
\end{array}\right] \\
& R_{y y}(\tau)=\sum_{i=1}^{n_{w}} \frac{1}{2 n_{\mathrm{r}} m} \sum_{j=0}^{2 n_{w} m-1} y_{s}^{i}(j+\tau)\left[y_{s}^{i}(j)\right]^{\mathrm{T}}, \\
& X=\sum_{I=1}^{n_{w}} \frac{1}{2 n_{\mathrm{r}} m} \sum_{j=0}^{2 n_{w} m-1} x_{s}^{I}(j+\tau)\left[x_{s}^{I}(j)\right]^{\mathrm{T}}, \\
& W_{q}=\left[\begin{array}{cccc}
W(0) & W(1) & \ldots & W(q-1) \\
W(1) & W(0) & \ldots & W(q-2) \\
\vdots & \vdots & \ddots & \vdots \\
W(q-1) & W(q-2) & \ldots & W(0)
\end{array}\right], \\
& W(\tau)= \begin{cases}\left(n_{\mathrm{r}}-\tau \frac{m-1}{m}\right) \text { block-diag }\left[a_{1}^{2}, \ldots, a_{n_{w}}^{2}\right], & \tau<n_{\mathrm{r}}, \\
0-n_{\mathrm{r}} \times n_{\mathrm{r}} \text { matrix, } & \tau \geq n_{\mathrm{r}},\end{cases}
\end{aligned}
$$


$a_{I}$ is the PRBS magnitude used for the Ith input and $\Delta_{q}$ has the following property based upon Theorem 3.1 in Ref. [6]:

$$
\lim _{m \rightarrow \infty}\left\|\Delta_{q}\right\|_{F}=0
$$

where $\|\cdot\|$ denotes Frobenius norm. Property of (III-14) is the key of allowing the use of the PRBS to develop a q-Markov Cover since for larger enough $m$,

$$
Q_{q} R_{q} Q_{q}^{\mathrm{T}}=Q_{q} O_{q} X O_{q}^{\mathrm{T}} Q_{q}^{\mathrm{T}}+Q_{q} H_{q} W_{q} H_{q}^{\mathrm{T}} Q_{q}^{\mathrm{T}}
$$

\section{III.B. Weighted Multirate q-Markov Cover Theorem}

Let the unknown (presumed nonlinear) system

$$
\begin{array}{r}
x(k+1)=f(x(k), w(k)), \\
y(k)=g(x(k), w(k)),
\end{array}
$$

be subjected to an input sequence $\{w(0), w(1), w(2), \ldots\}$ generating the output sequence $\{y(0), y(1), y(2), \ldots\}$. We say that (III-16) is $q$-identifiable if there exists a linear model of the form (III-1) that can reproduce the same output sequence $\{y(0), y(1), y(2), \ldots, y(q-1)\}$ in response to the same input sequence $\{w(0), w(1), w(2), \ldots, w(q-1)\}$. The following lemma (see Ref. [6] for a proof) provides the necessary and sufficient condition for $q$-identifiability.

LEMMA III-1 Let $R_{q}$ and $H_{q} W_{q} H_{q}^{\mathrm{T}}$ be constructed from the response of system (III-16) given the PRBS input $w$ with period $m=\infty$. Then the system (III-16) generating the data $y(i)(i=0,1,2, \ldots)$ is q-identifiable if and only if the data matrix $D_{q}=R_{q}-H_{q} W_{q} H_{q}^{\mathrm{T}} \geq 0$.

If the system generating the data is not q-identifiable, then it is possible for the q-Markov Cover identification to construct a least squares fit to the data with a linear model, see Refs. [1,2], but there exists no linear model of any order that can produce the data exactly.

The computation of the weighted q-Markov Covers starts from the data matrices $D_{q}, M_{q}=\left[H_{1}^{\mathrm{T}}, H_{2}^{\mathrm{T}}, \ldots, H_{q-1}^{\mathrm{T}}\right]^{\mathrm{T}}$, and $H_{0}$. Based upon Lemma III-1 it is concluded that a state realization $\{\hat{A}, \hat{B}, \hat{C}, \hat{H}\}$ is a 
q-Markov Cover of given matrices $D_{q}, M_{q}=\left[H_{1}^{\mathrm{T}}, H_{2}^{\mathrm{T}}, \ldots, H_{q-1}^{\mathrm{T}}\right]^{\mathrm{T}}$, and $H_{0}$ if the data matrices $\hat{D}_{q}, \hat{M}_{q}$, and $\hat{H}_{q}$ constructed from system matrices $\{\hat{A}, \hat{B}, \hat{C}, \hat{H}\}$ are identical to the data matrices $D_{q}, M_{q}$, and $H_{0}$, respectively. Define $P_{q}$ to be a full column rank square root of $Q_{q} D_{q} Q_{q}^{\mathrm{T}}$, i.e.,

$$
Q_{q} D_{q} Q_{q}^{\mathrm{T}}=Q_{q}\left(R_{q}-H_{q} W_{q} H_{q}^{\mathrm{T}}\right) Q_{q}^{\mathrm{T}}=P_{q} \cdot P_{q}^{\mathrm{T}},
$$

and denote $r$ to be the rank of $Q_{q} D_{q} Q_{q}^{\mathrm{T}}$. Partition matrix $P_{q}$ as

$$
P_{q}=\left[P_{0}^{\mathrm{T}}, P_{1}^{\mathrm{T}}, \ldots, P_{q-1}^{\mathrm{T}}\right], \quad P_{i} \in \mathfrak{R}^{n_{y} \times r}, i=0,1, \ldots, q-1 .
$$

Define

$$
P=\left[P_{0}^{\mathrm{T}}, P_{1}^{\mathrm{T}}, \ldots, P_{q-2}^{\mathrm{T}}\right], \quad \bar{P}=\left[P_{1}^{\mathrm{T}}, P_{2}^{\mathrm{T}}, \ldots, P_{q-1}^{\mathrm{T}}\right] .
$$

The following lemma, which extends the result in Ref. [3], transfers the q-Markov Cover problem to a linear algebra problem.

LEMma III-2 Assume that the period of the PRBS is infinity, i.e., $m=\infty$. A realization $\{A, B, C, H\}$ with $X$ equal to identity is a $q$-Markov Cover matching the data matrices $D_{q}, M_{q}$ and $H_{0}$ if and only if there exists realization $\{\hat{A}, \hat{B}, \hat{C}, \hat{H}\}$ which is a unitary coordinate transformation of $\{A, B, C, H\}$ such that the following equations are satisfied:

$$
\begin{aligned}
P[\hat{A}, \hat{D}] & =\left[\bar{P}, Q_{q-1} M_{q}\right], & {[\hat{A}, \hat{D}][\hat{A}, \hat{D}]^{\mathrm{T}}=I, } \\
\hat{C} & =Q^{-1} P_{0}, \quad \text { and } & \hat{H}=Q^{-1} H_{0}
\end{aligned}
$$

Using the linear algebra result for all solutions of (III-20) all q-Markov Cover can be generated from the given data matrices $Q_{q} R_{q} Q_{q}^{\mathrm{T}}$, $Q_{q-1}^{\mathrm{T}} M_{q}$, and $Q^{-1} H_{0}$.

THEOREM III-1 Suppose that the data matrices $Q_{q} R_{q} Q_{q}^{\mathrm{T}}, Q_{q-1}^{\mathrm{T}} M_{q}$, and $Q^{-1} H_{0}$ are generated by the steady state responses $y_{s}^{I}(k)$ $\left(I=1,2, \ldots, n_{w}\right)$ defined in (III-6) from the PRBS excitation with $m=\infty$. Then all minimal q-Markov Covers of the system with identity state covariance, data matrices $D_{q}, M_{q}$, and $H_{0}$ can be obtained by the 
following equations:

$[\hat{A}, \hat{D}]=P^{+}\left[\bar{P}, Q_{q-1} M_{q}\right]+V_{p} U V_{\bar{P}}^{\mathrm{T}} ; \quad \hat{C}=Q^{-1} P_{0}, \quad$ and $\quad \hat{H}=Q^{-1} H_{0}$,

where matrix $U$ is arbitrarily unitary with dimension $\left(r-r_{1}\right) \times\left(r-r_{1}\right)$ ( $r_{1}$ is the rank of $P$ ), $V_{p}$ is a column unitary matrix whose column space belongs to the null of $P$ with dimension $r \times\left(r-r_{1}\right)$, and $V_{p}$ is also column unitary, whose column space belongs to the null of $\left[\bar{P} Q_{q-1} M_{q}\right]$ with dimension $\left(r-n_{u}\right) \times\left(r-r_{1}\right)$. Furthermore, the q-Markov Covers (III-21) are stable and exist for any $q>0$.

The proof of Theorem III-1 follows the same procedure as the results in Refs. [3,6].

\section{III.C. Weighted Multirate q-Markov Cover Algorithm}

The results of Theorem III-1 may not sound interesting because the signal $y_{s}^{I}(k)\left(I=1,2, \ldots, n_{w}\right)$ defined in (III-6) and the PRBS with $m=\infty$ cannot be generated in practice. But Theorem III-1 suggests that the q-Markov Cover can be approximated using the PRBS with large enough period number $m$ because in this case Eq. (III-10) can be approximated by (III-15). Also, the steady state signal $y_{s}^{I}(k)$ $\left(I=1,2, \ldots, n_{w}\right)$ can be obtained approximately in the experiment by taking the steady state responses of system (III-16). Following is the weighted multirate q-Markov Cover algorithm using PRBS.

(1) Set parameters $m$ and $n_{\mathrm{r}}$ (ratio of PRBS sampling period over system output sampling one, see (III-7)), and then, conduct experiments to obtain steady state output responses $y_{s}^{I}(k)\left(I=1,2, \ldots, n_{w}\right)$.

(2) Compute autocorrelation matrices $R_{y y}(i)$ and cross-correlation matrices $R_{y w}(i)(i=0,1, \ldots, q-1)$ by the following:

$$
\begin{aligned}
& R_{y y}(i)=\frac{1}{2 m} \sum_{I=1}^{n_{w}} \sum_{k=0}^{2 n_{\mathrm{r}} m-1} y_{s}^{I}(k+i)\left[y_{s}^{I}(k)\right]^{\mathrm{T}}, \\
& R_{y w}(i)=\frac{1}{2 m} \sum_{I=1}^{n_{w}} \sum_{k=0}^{2 n_{r} m-1} y_{s}^{I}(k+i) u_{I}(k)\left[e_{I}\right]^{\mathrm{T}},
\end{aligned}
$$


where $u_{I}(k)\left(I=1,2, \ldots, n_{w}\right)$ is the PRBS signal for the $I$ th input and $y_{s}^{I}(k)\left(I=1,2, \ldots, n_{w}\right)$ is the steady state response of system (III-1) with only the $I$ th input is excited by $u_{I}(k)$. Then the system Markov matrices can be computed by

$$
H_{i}=R_{y w}(i) W^{-1},
$$

where matrix $W$ is defined in (III-13), and notice that in (III-13) $a_{I}$ $\left(I=1,2, \ldots, n_{w}\right)$ is the magnitude of the PRBS for the $I$ th input channel. See Ref. [6] for the derivation of Eqs. (III-22) and (III-23).

(3) Select parameter $q$, and form matrices $R_{q}, M_{q}$, and $H_{q}$ by

$$
\begin{gathered}
R_{q}=\left[\begin{array}{cccc}
R_{y y}(0) & R_{y y}(1) & \ldots & R_{y y}(q-1) \\
R_{y y}(1) & R_{y y}(0) & \ldots & R_{y y}(q-2) \\
\vdots & \vdots & \ddots & \vdots \\
R_{y y}(q-1) & R_{y y}(q-2) & \ldots & R_{y y}(0)
\end{array}\right], \\
M_{q}=\left[\begin{array}{c}
H_{1} \\
H_{2} \\
\vdots \\
H_{q-1}
\end{array}\right], \quad H_{q}=\left[\begin{array}{cccc}
H_{0} & 0 & \ldots & 0 \\
H_{1} & H_{0} & \ldots & 0 \\
\vdots & \vdots & \ddots & \vdots \\
H_{q-1} & H_{q-2} & \ldots & H_{0}
\end{array}\right]
\end{gathered}
$$

and $Q_{q}$ is a block diagonal matrix with $q$ blocks of positive definite matrix $Q$. Note that matrix $Q$ is selected by user to scale the output.

(4) Form matrix $P_{q}$ by

- Computing $D_{q}=Q_{q}\left[R_{q}-H_{q} W_{q} H_{q}^{\mathrm{T}}\right] Q_{q}^{\mathrm{T}}$, where $W_{q}$ is defined in (III-13).

- Doing the Schur decomposition of $D_{q}$ to obtain

$$
D_{q}=\left[\begin{array}{ll}
\hat{P}_{q} & \tilde{P}_{q}
\end{array}\right]\left[\begin{array}{cc}
\Lambda & 0 \\
0 & \tilde{\Lambda}
\end{array}\right]\left[\begin{array}{c}
\hat{P}_{q}^{\mathrm{T}} \\
\tilde{P}_{q}^{\mathrm{T}}
\end{array}\right]
$$

such that the diagonal elements of block-diag $\lfloor\Lambda \tilde{\Lambda}\rfloor$ are in decreasing order, and $\Lambda$ is chosen such that $\bar{\sigma}\|\tilde{\Lambda}\| \ll \underline{\Lambda}\|\Lambda\|$, 
where $\Lambda \in \mathfrak{R}^{r \times r}$, and $\bar{\sigma}\|M\|$ and $\underline{\sigma}\|M\|$ denotes the maximum and minimum singular values of $M$.

- Constructing $P_{q}$ by

$$
P_{q}=\hat{P}_{q} \Lambda^{1 / 2} .
$$

(5) Construct matrices $P, \bar{P}$, and $V_{p} U V_{p}^{\mathrm{T}}$ by

- Defining

$$
\begin{aligned}
& P_{q}^{\mathrm{T}}=\left\lfloor P_{0}^{\mathrm{T}}, P_{1}^{\mathrm{T}}, \ldots, P_{q-1}^{\mathrm{T}}\right\rfloor, \\
& P^{\mathrm{T}}=\left[P_{0}^{\mathrm{T}}, P_{1}^{\mathrm{T}}, \ldots, P_{q-2}^{\mathrm{T}}\right], \\
& \bar{P}^{\mathrm{T}}=\left[P_{1}^{\mathrm{T}}, P_{2}^{\mathrm{T}}, \ldots, P_{q-1}^{\mathrm{T}}\right] .
\end{aligned}
$$

- Making the singular value decomposition of $P$ and $\left[\tilde{P}, M_{q}\right]$

$$
\begin{aligned}
& P=\left[\begin{array}{ll}
U_{a} & U_{b}
\end{array}\right]\left[\begin{array}{cc}
\Sigma_{a} & 0 \\
0 & 0
\end{array}\right]\left[\begin{array}{c}
V_{a}^{\mathrm{T}} \\
V_{b}^{\mathrm{T}}
\end{array}\right], \\
& {\left[\bar{P}, M_{q}\right]=\left[\begin{array}{ll}
U_{c} & U_{d}
\end{array}\right]\left[\begin{array}{cc}
\Sigma_{c} & 0 \\
0 & 0
\end{array}\right]\left[\begin{array}{c}
V_{c}^{\mathrm{T}} \\
V_{d}^{\mathrm{T}}
\end{array}\right] .}
\end{aligned}
$$

- If $P$ is full rank, letting, $V_{p} U V_{\bar{p}}^{\mathrm{T}}=0$, and else,

$$
V_{p} U V_{\tilde{p}}^{\mathrm{T}}=V_{b} U V_{d}^{\mathrm{T}},
$$

where $U$ is an arbitrary unitary matrix.

(6) Compute $\hat{A}, \hat{D}, \hat{C}$ and $\hat{H}$ by

$$
\begin{aligned}
& \hat{A}=P^{+} \bar{P}+V_{p} U V_{\bar{p}}^{\mathrm{T}} I_{1}, \quad I_{1}=\left[\begin{array}{ll}
I_{r} & 0
\end{array}\right] ; \\
& \hat{D}=P^{+} M_{q}+V_{p} U V_{\bar{p}}^{\mathrm{T}} I_{2}, \quad I_{2}=\left[\begin{array}{ll}
0 & I_{n_{w}}
\end{array}\right] ; \\
& \hat{C}=Q^{-1} P_{0}, \quad \hat{H}=Q^{-1} H_{0} .
\end{aligned}
$$

\section{III.D. Matlab GUI and Selection of PRBS System Identification Parameters}

Subsection III.C describes the mathematical details of the multirate q-Markov system identification algorithm using PRBS. In practical application, in order to obtain a good system identification model, 


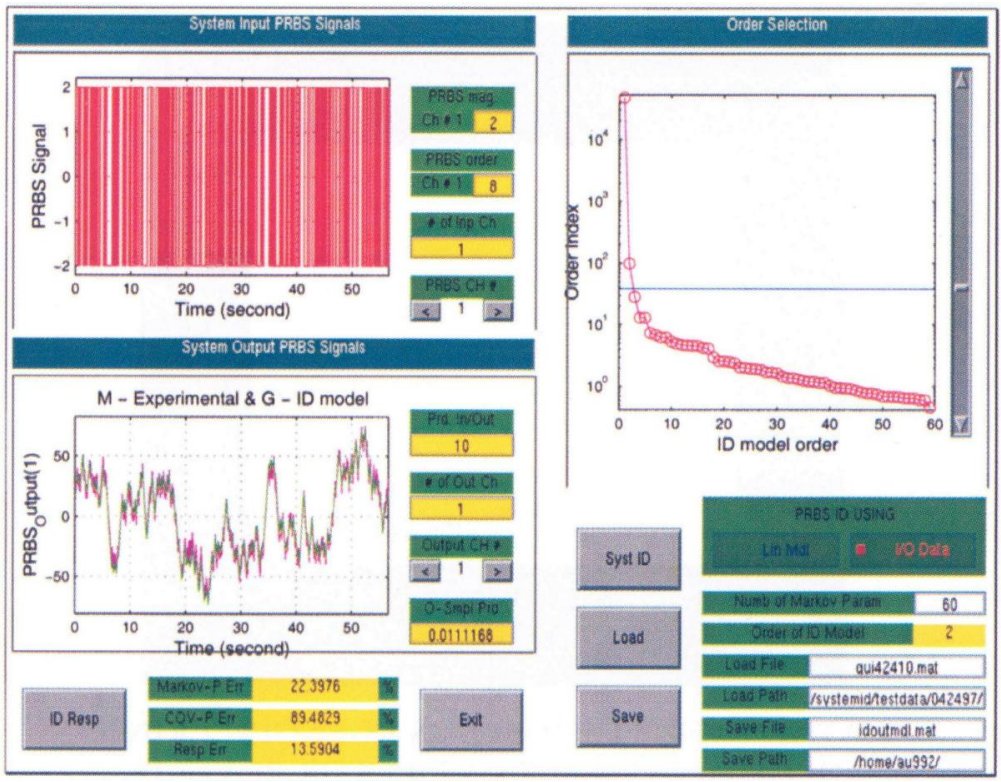

FIGURE 2 Weighted multirate PRBS system identification GUI. (See Color Plate II at the back of the issue.)

parameters like $q$ and $r$ defined in Step 4 of the algorithm may need to be tuned to have a good fit of the test data corresponding to the identified model. In order to make the system identification tuning process (varying parameters $q$ and $r$ ) easy, an Matlab GUI is developed. Figure 2 shows the Matlab system identification GUI.

The functionality of the PRBS system identification GUI is listed below:

- System input block (upper-left portion) specifies the input PRBS. The GUI allows the user to view number of input channels, PRBS magnitude and order for any selected input channel. The selected PRBS signal is also plotted on the GUI.

- System output block (middle-left portion) specifies and displays the system output response of a loaded data file. The GUI allows the user to view the number of output channels, output sample period, and the input/output sample period ratio $n_{\mathrm{r}}=T_{\mathrm{p}} / T$. 
- Loading/saving data file block (lower-right portion) allows the user to load a test data file for PRBS system identification and save the identified model into a specified data file. This block also make it possible to modify the selected identification parameter $q$ (see Step 1 of the PRBS system identification algorithm in Subsection III.C).

- Order selection block (upper-right portion) plots the diagonal elements of $\Lambda$ (see (III-25)). Note that each plotted point corresponding to one order of the identified model. Moving the slider on right allows the user to select parameter $r$ (see Step 4 of the PRBS system identification algorithm in Subsection III.C).

- System response block (lower-left portion) calculates the response error and plots the model response over the test response in the system output block. This provides the feeling of the system identification quality with selected system identification parameters $q$ and $r$.

The general procedure of weighted multirate system identification using PRBS and its parameter selection can be described in the following steps:

\section{Step 1: Design the PRBS system identification experiments}

To design PRBS system identification experiments, one needs to select the PRBS magnitudes $a_{i}\left(i=1,2, \ldots, n_{w}\right)$ and the order of PRBS corresponding to parameter $m$, the system output sample period $T$ and the integer sample ratio $n_{\mathrm{r}}=T_{\mathrm{p}} / T$, where $T_{\mathrm{p}}$ is the sample period for generating PRBS. In general, the magnitude of PRBS is selected to maximize the system output signal to noise ratio, and the order of the PRBS shall cover the dominated frequencies of the identified system such that these modes can be excited by PRBS. The sample period ratio is selected such that the actuator is able to respond to the PRBS command. For instance, consider the diesel engine system from the fuel command to the engine speed output. Note that in a diesel engine, fuel is directly injected into the individual cylinder at a proper crank angle, or in other words, the fuel injection is a discrete event. Identifying an engine model from the fuel command to the engine speed requires that the PRBS fuel excitation can be executed by the fuel system. For a six-cylinder four-stroke diesel engine running at $600 \mathrm{RPM}$, the fuel injection frequency is $30 \mathrm{~Hz}$. If the desired system output sample rate $(1 / T)$ is at $100 \mathrm{~Hz}$, the input PRBS excitation cannot be generated at $100 \mathrm{~Hz}$ since the PRBS fuel excitation cannot 
be executed by the fuel system. This requires a lower PRBS generation frequency $\left(1 / T_{\mathrm{p}}\right)$ than $30 \mathrm{~Hz}$ to make sure that the PRBS fuel command can be executed by the fuel system actuators. If the PRBS generation sample period is selected to $T_{\mathrm{p}}=0.1(\mathrm{~s})$, the selected sample ratio is $n_{\mathrm{r}}=T_{\mathrm{p}} / T=10$.

Step 2: Run the system identification test and collect experimental data After designing the system identification experiment, the next step is to run the test and collect the experimental data. From the derivation of the PRBS system identification theorem, steady state responses (defined in (III-6)) of one PRBS cycle ( $2 n_{\mathrm{r}} m$ points) are used for system identification. After the PRBS perturbation for a given channel is applied, the response data shall not be collected until the corresponding system responses are periodic and have the same period as the PRBS (this indicates that steady state has been reached). For the system with multiple input channels, the PRBS input excitation needs to be applied individually, and the system output responses $\left(y_{q}^{I}(k)\right.$ $\left(I=1,2, \ldots, n_{w}\right)$, see (III-6)), corresponding to each PRBS input channel shall be collected.

\section{Step 3: System identification using GUI}

After the experimental data is collected and converted into a Matlab data file ready to be loaded into Matlab, the PRBS system identification GUI can be used to obtain a linearized system model. To have a good system identification result, the following parameters need to be determined: output weighting matrix $Q$, number $q$ of Markov and covariance parameter to be preserved, and the identified system order $r$. In general, the output weighting matrix is used to balance the output response scale for different output channels. For example, if the magnitude of output channel one is ten times larger than channel two for a two-output system, the relative system identification error for output channel two could be bigger than channel one even though the absolute system identification error for both channels are the same. In this case, the output weighting matrix $Q$ can be used to adjust the output magnitude ratio for different output channels to improve relative modeling error for each individual output channel. The selection of parameter $q$ is related to how well the identified model responses match actual system ones. Increasing $q$ improves transient and steady state response match, but for a given PRBS order (given $m$ ) increasing 
$q$ introduces more relative system identification error due to the fact that finite PRBS order is used for system identification. Selection of model order parameter $r$ is described in Step 4 of the PRBS system identification algorithm. The good news is that the PRBS system identification GUI allows the user to select the parameters $q$ and $r$ and validate the identification quality through clicking the buttons on the GUI.

\section{AN APPLICATION TO DIESEL ENGINE SPEED CONTROL SYSTEM}

This section describes the application of the weighted multirate qMarkov Cover system identification to the diesel engine system from the engine fuel command to the engine speed output. The system identification is conducted using a Cummins $95 \mathrm{M} 11$ diesel engine in a test cell with PC controller (PC with extended I/O capable of controlling a diesel engine) instead of an ECM (Engine Control Module). The PC controller provides flexibility of implementing new control logic. The PRBS system identification software was implemented into the PC controller for experiment purpose.

The purpose of this system identification is to obtain a proper linearized engine model for model-based control design, especially, for the engine speed control system. In general, engine output torque is controlled by the fuel quantity injected into the individual cylinder, and the other engine control input such as engine timing (start of injection) affects the engine torque output a little which will be treated as disturbance input. An engine speed control system can be simplified in Fig. 3, where engine timing and load are treated as disturbance. The subsections below follow the system identification procedure defined in the previous section.

\section{IV.A. Design the PRBS System Identification Experiments}

The system identification experiments is design based upon rules stated in Step 1 of the system identification process in Subsection III.D. Selection of parameters $a_{1}$ (PRBS magnitude), $m=2^{n}-1$ (where $n$ is the order of PRBS), $T$ (system output sample period), and $T_{\mathrm{p}}$ (PRBS generation sample period) is shown in Table II. 


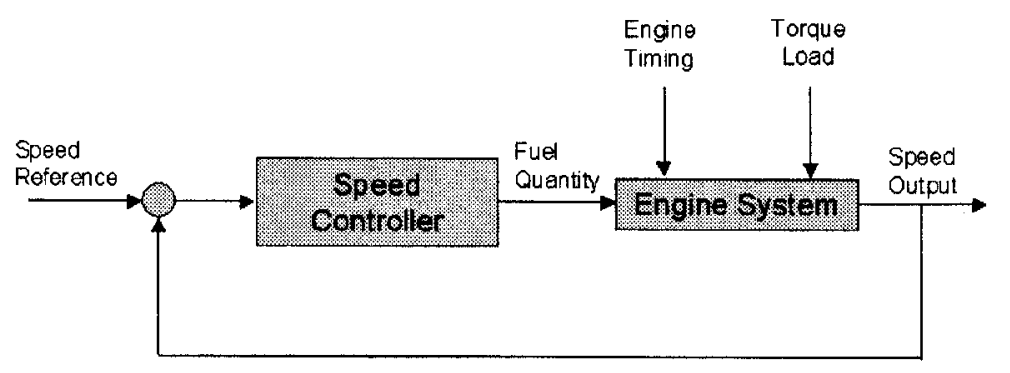

FIGURE 3 Engine speed control system.

TABLE II Parameters for PRBS system identification experiment

\begin{tabular}{|c|c|c|}
\hline Parameter & Selected values & Why \\
\hline$a_{1}$ & $2\left(\mathrm{~mm}^{3} /\right.$ stroke $)$ & $\begin{array}{l}2 \mathrm{~mm}^{3} / \text { stroke fueling fluctuation } \\
\text { may generate about } 50 \mathrm{RPM} \text { speed } \\
\text { variation for a bared engine. }\end{array}$ \\
\hline$m$ & $255(n=8)$ & $\begin{array}{l}\text { With selected } T \text { and } T_{\mathrm{p}} \text { the period of } \\
\text { PRBS in continuous time domain } \\
\text { is } 51 \mathrm{~s}\left(2 \times m \times T_{\mathrm{p}}\right) \text { which shall } \\
\text { be able to excite the system dominated } \\
\text { mode as low as } 0.025 \mathrm{~Hz} \text {. }\end{array}$ \\
\hline$T$ & $0.01(s)$ & $\begin{array}{l}T \text { is selected based upon the controller } \\
\text { sample rate (control algorithm executed } \\
\text { at about } 100 \mathrm{~Hz} \text { in PC controller). }\end{array}$ \\
\hline$T_{\mathrm{p}}$ & $0.1(\mathrm{~s})$ & $\begin{array}{l}T_{\mathrm{p}} \text { is selected such that the engine fuel } \\
\text { system is able to execute the PRBS fuel } \\
\text { command over } 600 \mathrm{RPM} \text {. Note that over the } \\
\text { given engine speed range, the lowest engine } \\
\text { fuel injection frequency (at } 600 \mathrm{RPM} \text { ) is } 30 \mathrm{~Hz} \\
\text { for a six-cylinder four-stroke engine. The PRBS } \\
\text { generation sample period is selected three } \\
\text { times slower than fuel injection period. }\end{array}$ \\
\hline
\end{tabular}

\section{IV.B. Experiment Setup and Data Collection}

The experiment used a bare M11 engine (engine is not connected to output) with a PC engine controller. The experiment setup (see Fig. 4) and data collection process can be described as follows: (a) default engine timing is used for this test, (b) engine DC fueling is used to run the engine at desired engine speed, (c) after engine speed is stable PRBS fuel excitation is added to perturb the engine system, and finally, (d) after the engine reaches its steady state corresponding to 


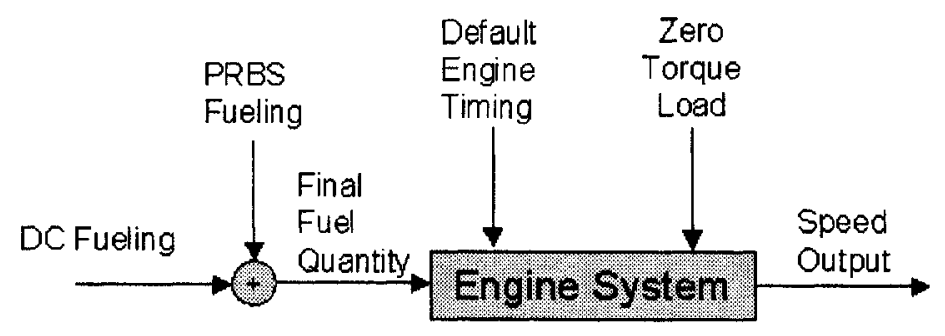

FIGURE 4 Experiment setup.

the PRBS perturbation, one PRBS cycle of the input PRBS perturbation and output engine speed response are collected at sample rate $1 / T$ (about $100 \mathrm{~Hz}$ ). Note that each collected data file is associated to a given engine speed. Therefore, the identified engine model is a function of the engine operational speed.

Note that due to engine friction torque fluctuation, holding a constant engine speed with constant fueling is not always feasible. In fact, closed-loop system identification (with a stable engine speed controller in the loop) is recommended. In this case, the speed controller holds the engine at the desired speed when the PRBS fueling disturbance is applied, and the identified model contains both engine and speed controller dynamics. The engine system model can be obtained by subtracting the controller dynamics. Both open- and closed-loop system identification were conducted and similar results are obtained. For this paper, only the open-loop system identification results are presented.

Many system identification tests were conducted, but only two system identification tests are described in this paper. That is, the system identification test at engine speed equal to 1150 and 1500 RPM. Figure 5 shows both the PRBS fueling disturbance in $\mathrm{mm}^{3} /$ stroke (upper plot) and the corresponding engine speed fluctuation in RPM (gray line of lower plot). In the system identification experiments, due to the PC control operational system the actual system sample period is about $0.0113 \mathrm{~s}$, leading one PRBS period equals to $57.6 \mathrm{~s}$. One of the important aspect during the system identification tests is judging if the collected system response is at the steady state or not. An easy way is using the inverse symmetric property $(u(k)=-u(k+m))$ of the inverse PRBS signal. Therefore, at the steady state the system output shall 


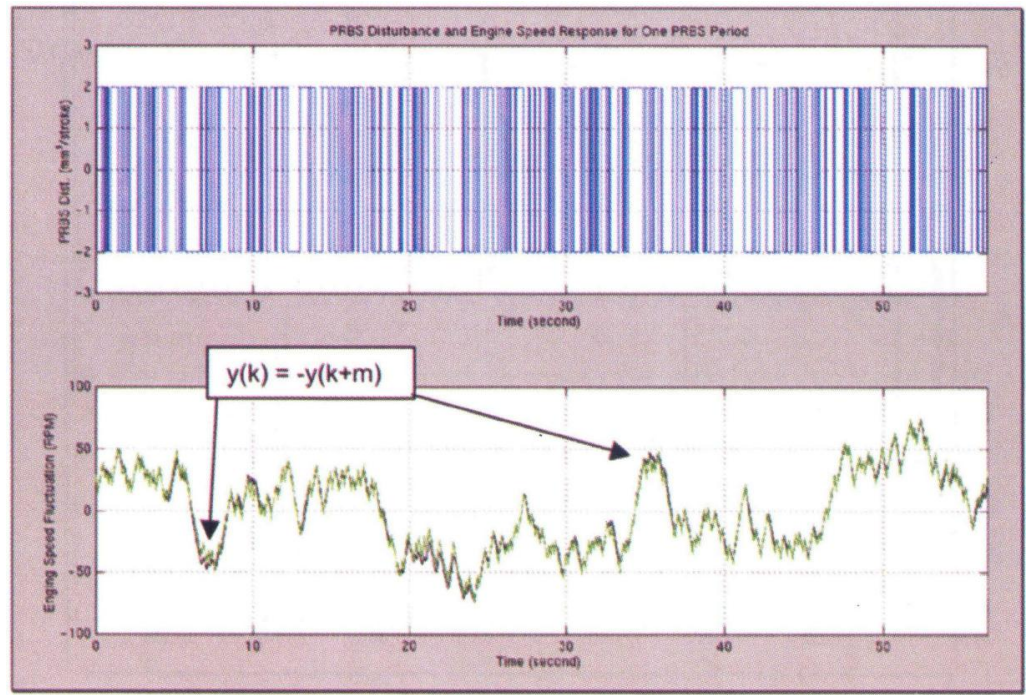

FIGURE 5 PRBS disturbance and corresponding engine speed response. (See Color Plate III at the back of the issue.)

have the similar property, that is,

$$
y(k)=-y(k+m)
$$

see the two points marked by arrows in Fig. 5. With the help of this technique, the author was able to collect the system identification data successfully without repeating any experiment.

\section{IV.C. System Identification Using Weighted Multirate q-Markov Cover}

The PRBS system identification GUI in Fig. 2 was used to obtain a linear engine model at a given engine speed. Step 3 of the procedure described in Subsection III.D was used for obtaining discrete time linear models. Since the system is an SISO one, the output weighting matrix is set to one. The number of Markov and covariance parameters to be preserved is selected to be 60 . That is, the first 60 Markov 
and covariance parameters of the identified model are close to these calculated ones based on (III-23) and (III-24). For practical purposes, the parameter $q$ is upper limited by the numerical accuracy of q-Markov Cover and the constraint $q \ll m$ due to a finite PRBS period. On the other hand, small $q$ may cost the accuracy of the identified model. The selected $q$ is based upon the concerns mentioned above, and especially, the accuracy of the identified model quality, such as how well the identified model response matches with the test one.

The order of the identified model is determined using the order selection portion (see Fig. 6) of the system identification GUI in Fig. 2. From Fig. 6 we can see that the largest gap exists between the first (top) dots and second one. This indicates that there exists one dominated first-order dynamics. For the diesel engine system from fuel command to engine speed, this dominated mode is obviously the dynamics from engine torque to engine speed. The second largest gap is between second and third modes. The identified model order was selected to be two since a third order model does not improve the identified model accuracy and the gap between second and third modes is the second largest. The steady state response of the identified engine

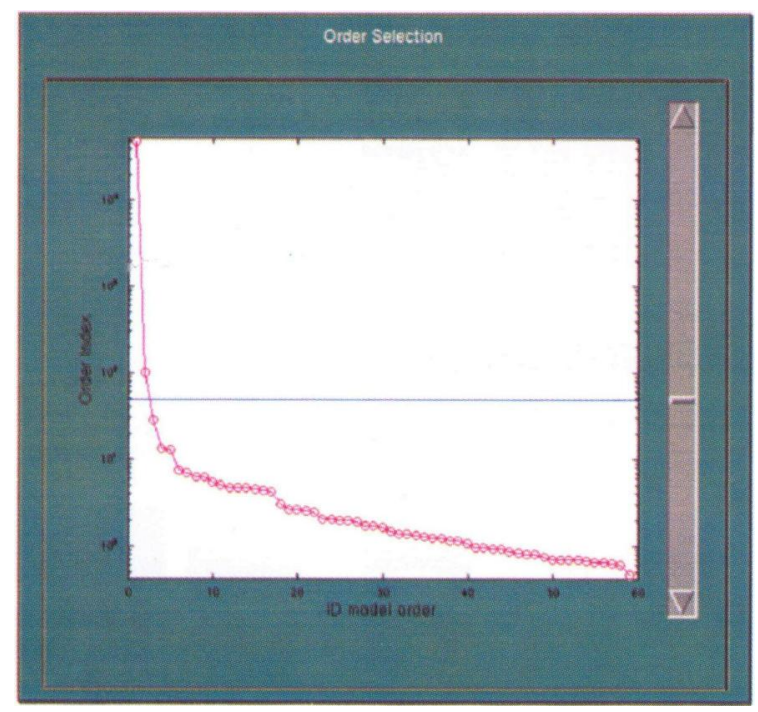

FIGURE 6 Model order selection. (See Color Plate IV at the back of the issue.) 
TABLE III Identified engine models

\begin{tabular}{lcc}
\hline Engine speed & $1150(\mathrm{RPM})$ & $1500(\mathrm{RPM})$ \\
\hline $\begin{array}{l}\text { Discrete transfer } \\
\text { function }\end{array}$ & $\begin{array}{c}\left(0.0198 z^{2}-0.1949 z+0.1562\right) / \\
\left(z^{2}-1.834 z+0.835\right)\end{array}$ & $\begin{array}{c}\left(0.0567 z^{2}-0.1888 z+0.2297\right) / \\
\left(z^{2}-1.732 z+0.7335\right)\end{array}$ \\
$\begin{array}{c}\text { Continuous } \\
\text { transfer function }\end{array}$ & $\begin{array}{c}114 \times\left(3.2 \times 10^{-5} s^{2}-0.0159 s+1\right) \\
((s / 0.348+1)(s / 15.88+1))\end{array}$ & $\begin{array}{c}85.6 \times\left(6.4 \times 10^{-5} s^{2}-0.0147 s+1\right) / \\
((s / 0.383+1)(s / 26.95+1)) \\
\text { Output sample } \\
\text { period }\end{array}$ \\
$\begin{array}{c}\text { PRBS generation } \\
\text { period }\end{array}$ & 0.0113 & 0.0113 \\
$\begin{array}{c}\text { Selected model } \\
\text { order } r\end{array}$ & 0.113 & 0.113 \\
Selected parameter $q$ & 2 & 2 \\
\hline
\end{tabular}

model with the same PRBS excitation as experimental one is drawn in the lower plot of Fig. 5 (black line). One can see that both responses are very close, indicating good system identification model.

Table III shows the two identified engine models for engine running at 1150 and 1500 RPM in both discrete and continuous time domains. The identified models are in discrete state space format, and then, transferred into the discrete transfer function format and continuous transfer function assuming zero order sampling. Consider the continuous time transfer function for engine running at $1150 \mathrm{RPM}$. The quadratic coefficient of the transfer function numerator is very close to zero. By setting it to zero we can rewrite the transfer function into the following format:

$$
G(s)=114 \times \frac{1-s / 62.9}{1+s / 15.88} \times \frac{1}{1+s / 0.348} .
$$

It is clear that the transfer function consists of two first-order dynamic components. The first portion of dynamics $(1-s / 62.9) /(1+s / 15.88)$ is a non-minimum phase transfer function approximating the pure delay dynamics due to the diesel engine fuel system, and the second portion of dynamics $1 /(1+s / 0.348)$ represents the engine mechanical dynamics from engine combustion torque to engine speed. From Table III one can see that the engine model DC gain decreases as the engine speed increases due to the quadratic increment of the engine friction torque. For the same reason, the time constant of the mechanical dynamics increases as engine speed. Figure 7 shows the step response of the 


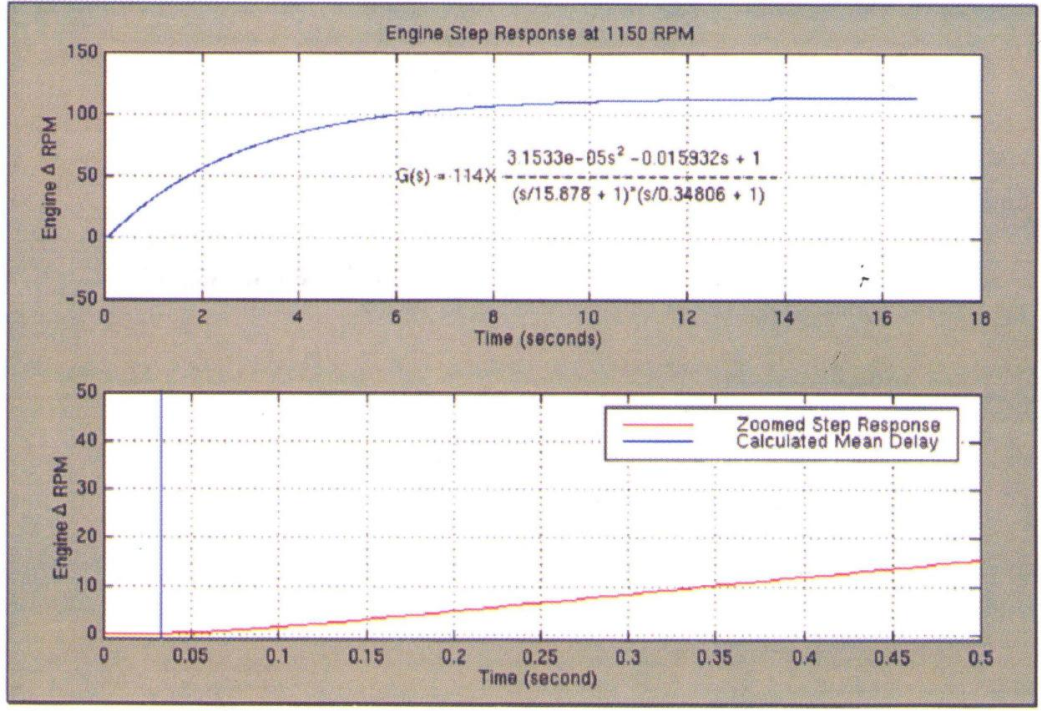

FIGURE 7 Step response and delay of identified model. (See Color Plate V at the back of the issue.)

engine transfer function at 1150 RPM. The lower plot of Fig. 7 shows a blow-up of the step response at the first $0.5 \mathrm{~s}$, where the vertical line indicated the calculated fuel system delay at 1150 RPM and given fuel command. We can see it clearly that identified transfer function provides almost identical time delay. This indicates that the PRBS system identification is able to use a first-order transfer function with nonminimum phase numerator to represent a pure time delay dynamics.

As a summary of this section, the PRBS q-Markov Cover is successfully applied to a diesel engine system from the fuel command to the engine speed output. The identified transfer functions have order of two. The pure time delay of the diesel fuel system is approximated by a first-order transfer function with a non-minimum phase numerator. The identified engine model is a function of engine speed due to nonlinear engine friction torque and fuel system delay. The identified engine model was used for designing engine idle speed governor using the OCC (Output Covariance Constraint, see Ref. [7]) control design method. The designed controller stabilizes the engine speed with proper response and over/under shoot performance at the first try. 


\section{v. CONCLUSIONS}

This paper successfully extend the PRBS q-Markov Cover system identification technique to a weighted multirate case. The extension allows the construction of a discrete time state space model at system output sample rate while the PRBS excitation sample rate is slower than the output one. The PRBS q-Markov Cover is successfully applied to a diesel engine system from the fuel command to the engine speed. The identified engine model is a function of engine speed due to a nonlinear engine friction torque and fuel system delay. The identified engine model was used for designing a engine idle speed controller using the OCC Ref. [7] control design method. The designed controller stabilizes the engine speed with proper response and over/under shoot performance at the first try. This provides confidence of using identified engine models for engine governor design.

\section{References}

[1] R.E. Skelton and B.D.O. Anderson, Q-Markov covariance equivalent realization, International Journal of Control, 53(1), 1986.

[2] A.M. King, U.B. Desai and R.E. Skelton, A generalized approach to q-Markov covariance equivalent realizations of discrete systems, Automatica, 4, 1998.

[3] K. Liu and R.E. Skelton, Identification and control of NASA's ACES structure, Proceedings of the American Control Conference, Boston, Massachusetts, USA, 1991.

[4] W.W. Perterson, Error Correcting Coding, MIT Technical Press, Cambridge, Massachusetts, USA, 1961.

[5] K.R. Godfrey, Correlation methods, Automatica, 3, 1980.

[6] G.G. Zhu, R.E. Skelton and P. Li, Q-Markov Cover identification using pseudorandom binary signals, International Journal of Control, 62(1), 1995, 1273-1290.

[7] G.G. Zhu, M A. Rotea and R.E. Skelton, A convergent algorithm for the output covariance constraint control problem, SIAM Journal on Control and Optimization, 35(1), 1997, 341-361. 


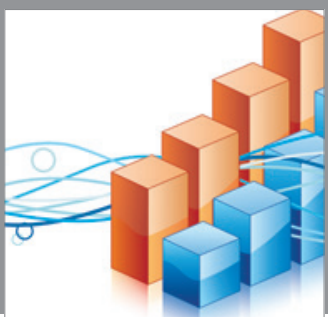

Advances in

Operations Research

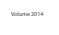

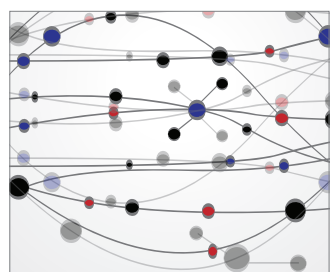

\section{The Scientific} World Journal
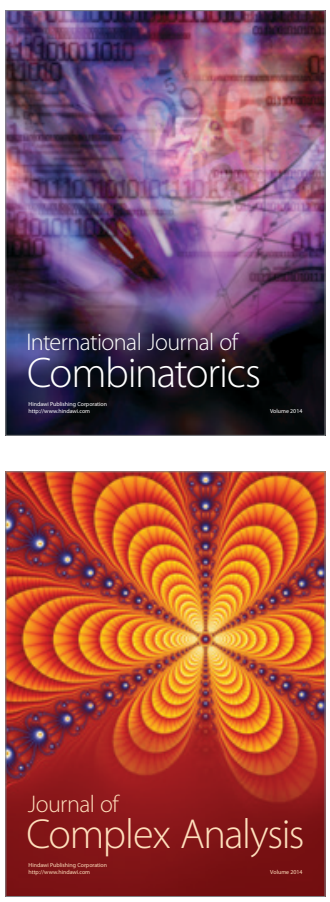

International Journal of

Mathematics and

Mathematical

Sciences
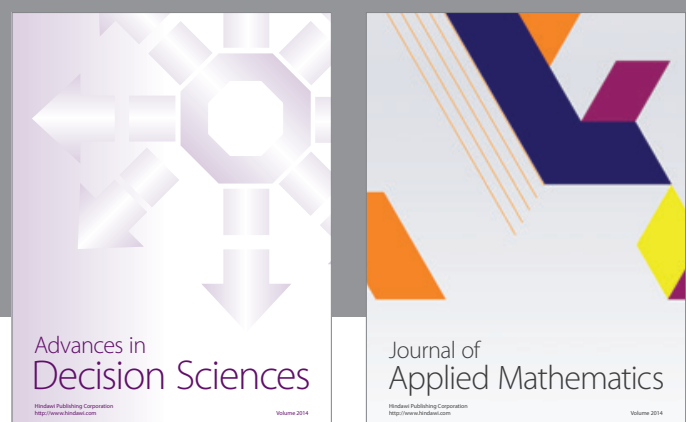

Journal of

Applied Mathematics
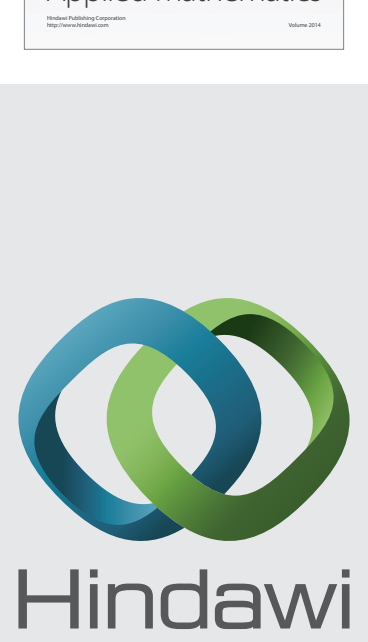

Submit your manuscripts at http://www.hindawi.com
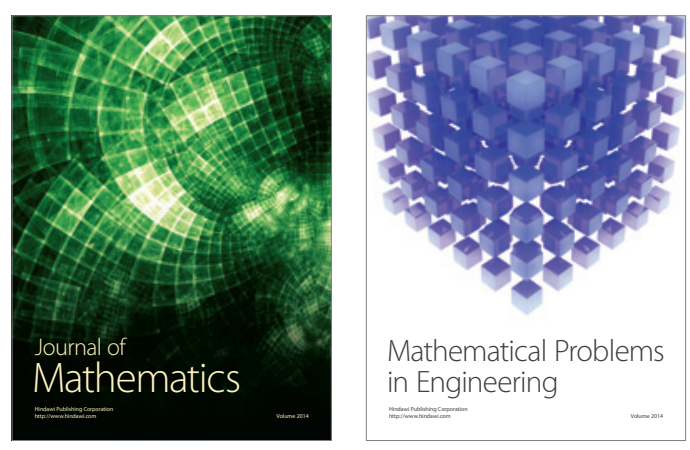

Mathematical Problems in Engineering
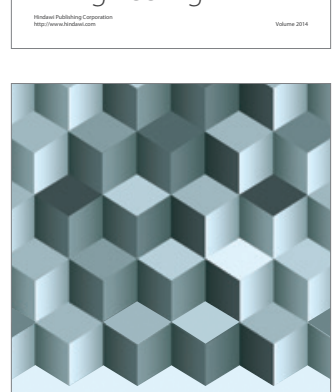

Journal of

Function Spaces
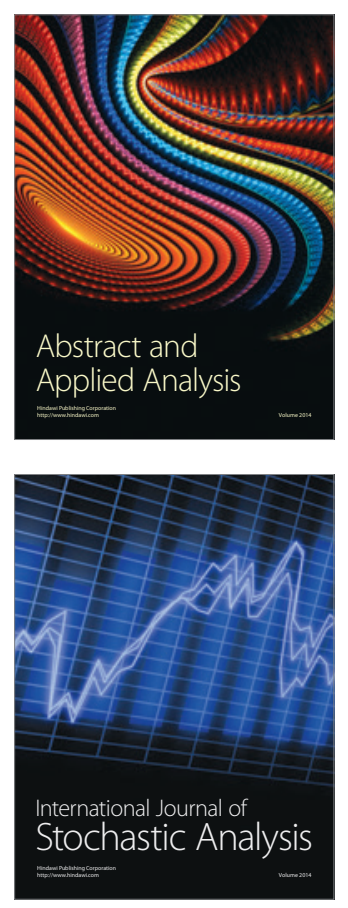

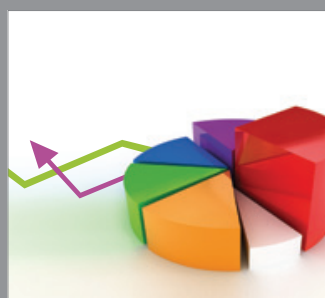

ournal of

Probability and Statistics

Promensencen
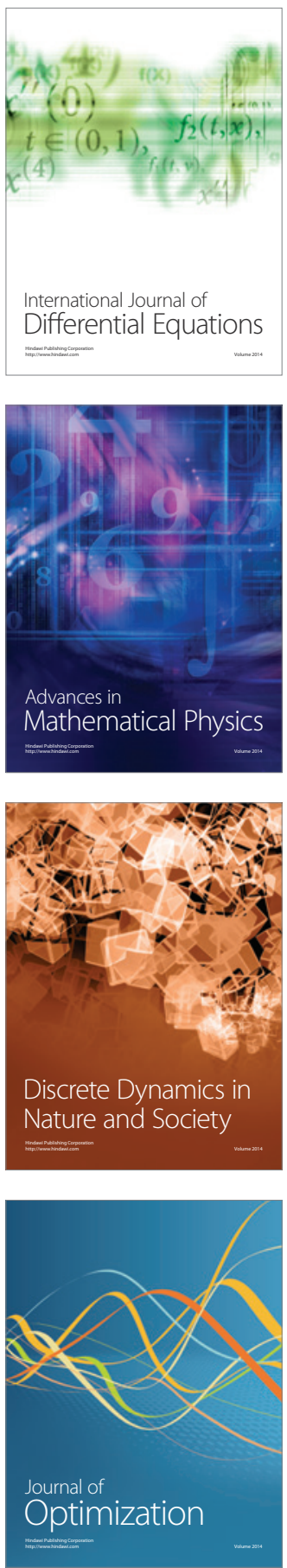